\title{
A STATISTICAL PROJECT CONTROL TOOL FOR
}

\section{ENGINEERING MANAGERS}

$2^{\text {nd }}$ International Workshop on Engineering Management

for Applied Technology

Joe C. Thompson Conference Center

Austin, TX

GARLAND T. BAUCH, PH. D.

INDUSTRIAL ENGINEERING

(SYSTEMS ENGINEERING/ENGINEERING MANAGEMENT)

NASA - JOHNSON SPACE CENTER

SPACE SHUTTLE DIVISION

SR\&QA OFFICE

AUGUST 16, 2001 


\section{AGENDA}

- Introduction

- Literature Review

- Problem Statement

- Research Methodology

- Research Results and Discussion

- Limitations of Study

- Contribution to the Body of Knowledge

- Future Research

- Conclusions 


\section{INTRODUCTION}

- Use of Projects Increasing Meredith (1988), Badiru, (1991), Kharbanda \& Pinto (1996), Pinto \& Kharbanda (1996), and Shenhar, Levy, \& Dvir (1996).

- Project Failures Increasing Globally

Badiru (1995), Balachandra (1989), Gioia (1996), Morris (1988), Morris and Hough (1987), and Tishler, Dvir, Shenhar, and Lipovetsky (1996)

- Application to Project Management 


\section{LITERATURE REVIEW}

- Project Success Definition

- Project Success Factors

- Project Control Tools (networking)

- Performance Measurement

- Statistical Process Control

- Continuous Assessment of Performance

- Statistical Project Control

- General Results 


\title{
LITERATURE REVIEW PROJECT SUCCESS DEFINITION
}

\author{
A Project Is Successful When the Cost, \\ Schedule, Technical Performance, and \\ Quality Satisfy the Customer.
}




\section{LITERATURE REVIEW PROJECT SUCCESS FACTORS}

- Project Goals, Definition, \& Mission

- Cost, Finance, \& Schedule

- Technical Uncertainty \& Performance

- Customer Satisfaction \& Acceptance

- Environmental, Social, \& Political Pressure

- Managerial \& Organizational Factors

- Communications

[Morris (1988), Slevin \& Pinto (1986), Tishler et. al (1996), Baker et. al. (1986), \& Woodard (1988)] 


\section{LITERATURE REVIEW \\ TRADITIONAL PROJECT CONTROL TOOLS}

- Gantt Charts

Gantt (1911)

- WBS

Kelley and Walker (1959)

- $\mathrm{CPM}$

Du Pont (1950s)

- PERT

Navy Polaris (1958) 


\section{LITERATURE REVIEW}

PERFORMANCE MEASUREMENT

- Actual Cost vs. Earned Value

- Planned (Budget) vs. Earned Value

- Technical Performance

- Required for > \$25 Million

[Project Management Guide, JSC 61100, NASA-JSC] 


\section{LITERATURE REVIEW \\ STATISTICAL PROCESS CONTROL}

- Shewhart Control Charts 1924

[Emerson \& Naehring (1985)], [Montgomery (1985)], [Johnson's Miller \& Freund (1994)]

- SQC Training/Deming, Et. Al.

[Emerson \& Naehring (1985)]

- Manufacturing Processes

- Tool Wear Model

[Banks (1989), Grant (1952), McClave and Benson (1994)] 


\section{LITERATURE REVIEW \\ CONTINUOUS ASSESSMENT OF PROJECT \\ PERFORMANCE (CAPP)}

- Predictive Tools Task Force (1992)

- Quantitative Real-time Data Collected

- Questionnaire Used 


\section{LITERATURE REVIEW STATISTICAL PROJECT CONTROL}

- No Literature Found 


\section{LITERATURE REVIEW GENERAL RESULTS}

- Existing Tools Mostly Empirical \& Subjective Slevin \& Pinto (1986)

- Need for Better Dynamic Tools

Balachandra \& Raelin (1984), Christian (1993), Goldin (1998), Meredith (1988), Morris (1988), \& Tadisina (1986)

- Consider Environmental Impact

Might \& Fisher (1985)

- Consider Customer Needs

Lipovetsky, Tishler, Dvir, \& Shenhar (1997) 


\section{PROBLEM STATEMENT}

- Resources Becoming More Limited

- Projects Are Increasing in Numbers

[Meredith (1988)]

- Project Failure Is Increasing

[Morris (1989)]

- Systematic Methods Needed

[Pinto \& Slevin (1988)]

- Existing Methods Limited 


\title{
OBJECTIVE
}

\author{
Provide a New
}

Statistical Project Control

Tool For

Project Managers 


\section{RESEARCH METHODOLOGY}

- Data Search/Collection

- Research Population

- Data Transformation

- Validation Steps

- Comparison of SPC and SPCT Charts 


\section{RESEARCH METHODOLOGY DATA SEARCH/COLLECTION}

- NASA

- Military

- Consultants

- Professional

- Commercial

- Institutes 


\section{RESEARCH METHODOLOGY RESEARCH POPULATION}

- Construction Industry Projects

- 17 Companies

- 54 Projects

- 76 Variables 


\section{RESEARCH METHODOLOGY DATA TRANSFORMATION}

- Traditional Control Chart Features

- Compute Normalized Time and Variables

- Pattern Analysis Rules

- Validation Steps

- Comparison of SPC and SPCT Charts 


\section{RESEARCH METHODOLOGY Traditional Control Chart Features}

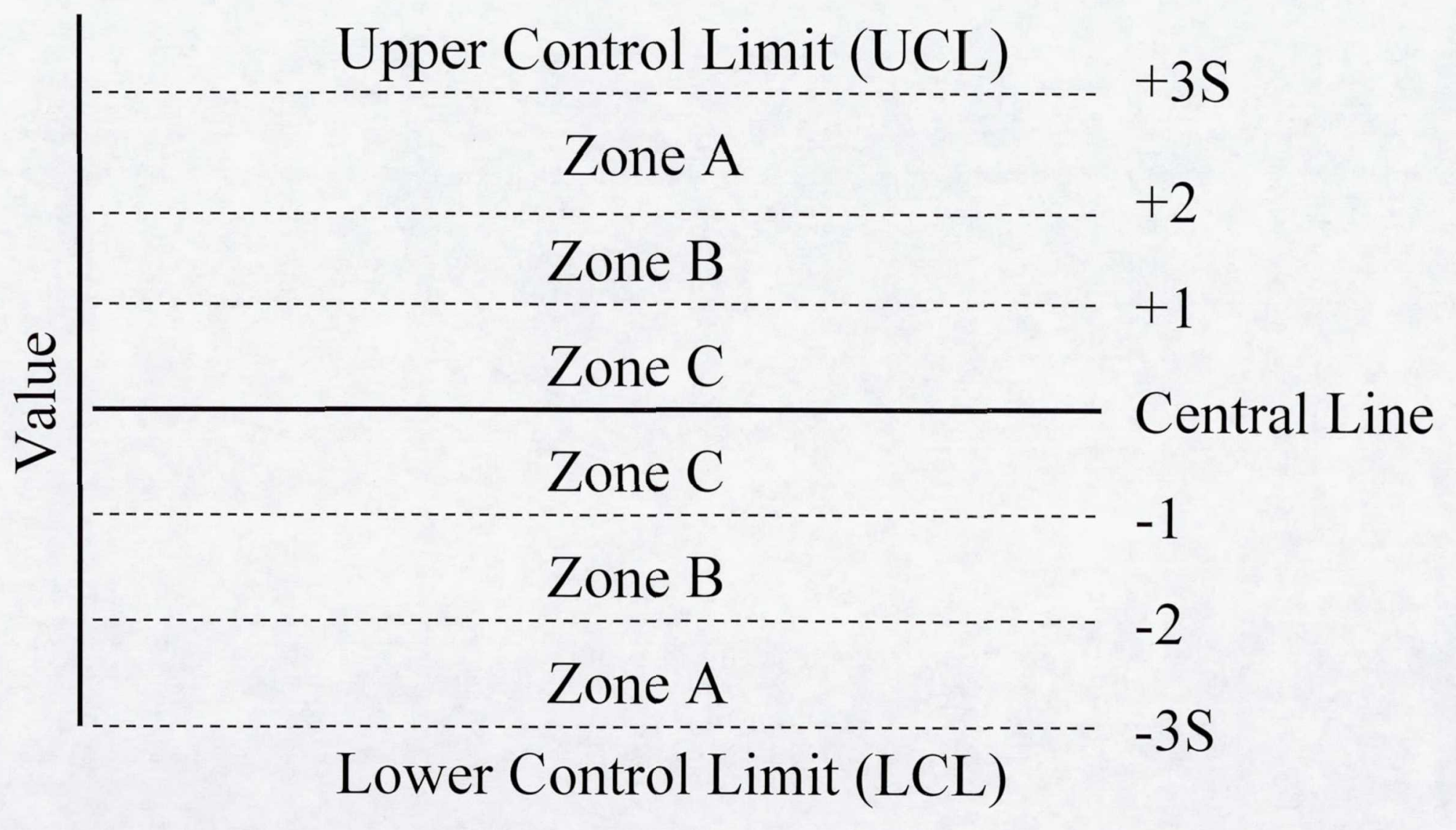

[McClave \& Benson (1994)] 


\section{RESEARCH METHODOLOGY DATA TRANSFORMATION COMPUTE NORMALIZED TIME}

Normalized

Original 


\section{RESEARCH METHODOLOGY DATA TRANSFORMATION COMPUTE NORMALIZED VARIABLE}

- Ratio

- Moving Average

- Exponential Smoothing

- \% Cumulative (Cum)

- Cum \% Cum

- Average Cum \% Cum 


\section{RESEARCH METHODOLOGY DATA TRANSFORMATION SPCT CHART}




\section{RESEARCH METHODOLOGY DATA TRANSFORMATION TRADITIONAL PATTERN ANALYSIS RULES}

Rule 1: 1 point beyond Zone A

Rule 2: 9 points in a row in Zone $\mathrm{C}$ or beyond

Rule 3: 6 pts. in a row steadily incr. or decreasing

Rule 4: 14 points in a row alternating up and down Rule 5: 2 out of 3 pts. in a row in Zone A or beyond Rule 6: 4 out of 5 pts. in a row in Zone B or beyond

[McClave \& Benson (1994)] 


\section{RESEARCH METHODOLOGY DATA TRANSFORMATION MODIFIED PATTERN ANALYSIS RULES}

- Rule 1m: 1 pt. beyond Zone A

Except for a slight exceeding in cost or design early in the project life cycle

- Rationale

-Initial start-up costs large sometimes

-Zero or low variable values

- Low values cause narrow control limits

-Insufficient trending data 


\section{RESEARCH METHODOLOGY DATA TRANSFORMATION MODIFIED PATTERN ANALYSIS RULES}

- Rule $2 \mathrm{~m}: 9$ pts. in a row in Zone $\mathrm{C}$ or beyond Except below the CL early in the project life cycle for expenditures or construction and late in the life cycle for design.

- Rationale

-Expenditures and construction are historically low early in the project life cycle

-Design is historically low late in the life cycle 


\section{RESEARCH METHODOLOGY DATA TRANSFORMATION MODIFIED PATTERN ANALYSIS RULES}

- Rule $3 \mathrm{~m}_{1}: 6$ pts. in a row dec. rel. to the $\mathrm{CL}$ Except for design during the latter part of the project life cycle.

- Rationale

Design historically decreases late in the project life cycle 


\section{RESEARCH METHODOLOGY DATA TRANSFORMATION MODIFIED PATTERN ANALYSIS RULES}

- Rule $3 \mathrm{~m}_{2}: 6$ pts. in a row inc. rel. to the CL Except for design during the early part of the project life cycle.

- Rationale

Design historically increases early in the project life cycle 


\title{
RESEARCH METHODOLOGY Comparison of SPC and SPCT
}

\author{
TRADITIONAL PROJECT
}




\section{RESEARCH RESULTS \\ Chart Types}

- Actual Owner Expenditure

- Actual \% Design Complete

- Actual \% Construction Complete

- Actual Cost of Change Orders 


\section{RESEARCH METHODOLOGY QUANTITATIVE VALIDATION STEP}

- Plot 3 Different Successful Projects Does Not Violate Pattern Analysis Rules

- Plot 3 Different Failed Violates Pattern Analysis Rules

- Success/Failure Defined By Owner 


\section{RESEARCH RESULTS SPCT Validation Using Successful Project}

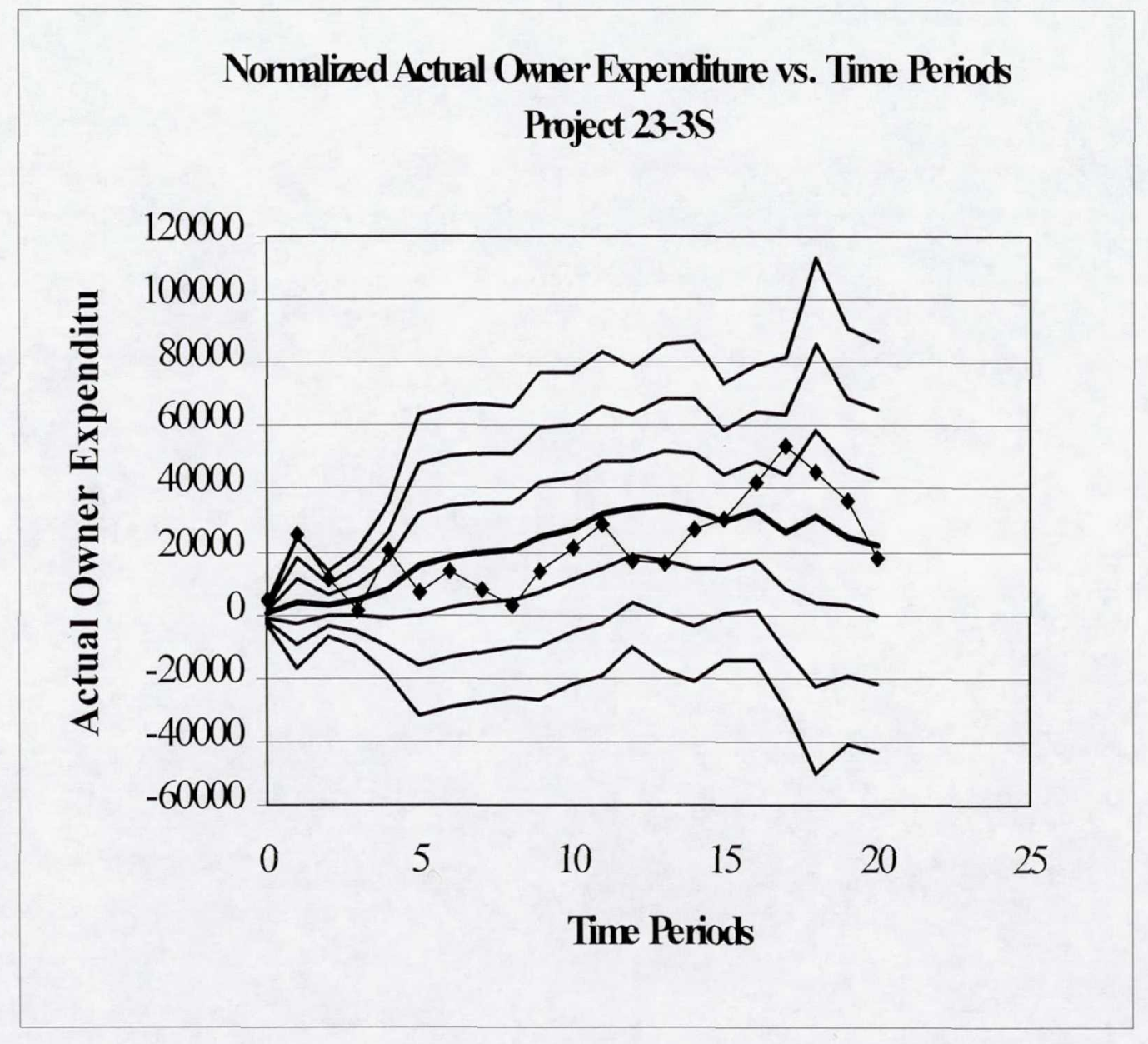

- Process industry

- In-control per Rules $1 \mathrm{~m} \& 2 \mathrm{~m}$

- Pattern validates control chart

\begin{tabular}{lllll}
$\frac{\mathrm{TP}}{0}$ & $\underline{\mathrm{LCL}}$ & $\frac{\mathrm{CL}}{2798}$ & $\mathrm{UCL}$ & $23-3 \mathrm{~S}$ \\
\hline & 271 & 3340 & $\frac{450}{450}$
\end{tabular}

$\begin{array}{lllll}5 & -31912 & 15862 & 63636 & 743\end{array}$

$\begin{array}{llllll}10 & -21891 & 27427 & 76744 & 21432\end{array}$

$\begin{array}{llllll}15 & -14300 & 29648 & 73597 & 30533\end{array}$

$\begin{array}{lllll}20 & -43350 & 21872 & 87094 & 18079\end{array}$ 


\section{RESEARCH RESULTS \\ SPCT Validation Using Failed Project}

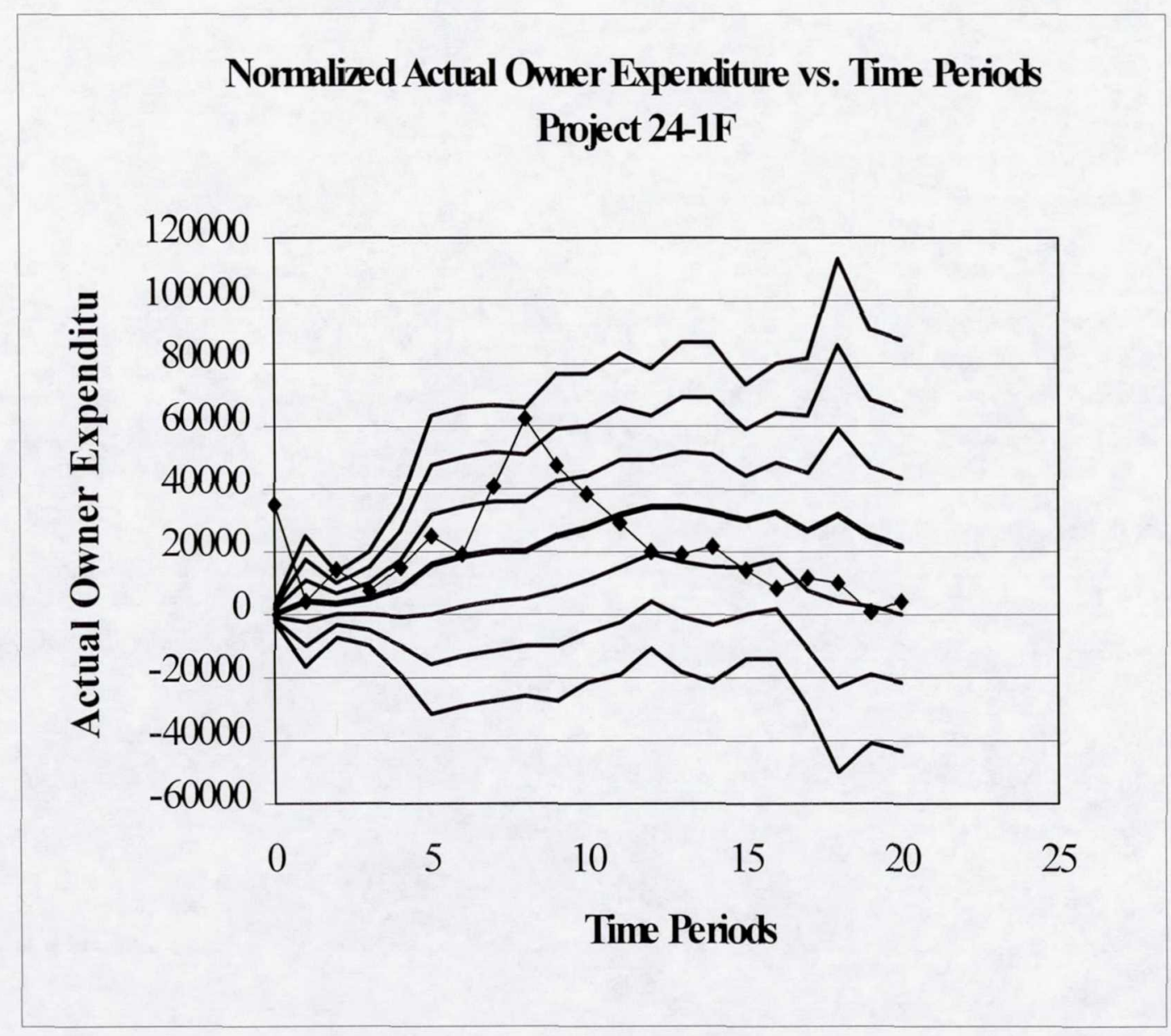

-Process industry

- Out-of-control per Rules 1, 2, \& 3

- Pattern validates control chart

\begin{tabular}{|c|c|c|c|c|}
\hline TP & $\underline{\mathrm{LCL}}$ & CL & UCL & 24-1F \\
\hline 0 & -2798 & 271 & 3340 & 34769 \\
\hline 5 & -31912 & 15862 & 63636 & 25265 \\
\hline 10 & -21891 & 27427 & 76744 & 38152 \\
\hline 15 & -14300 & 29648 & 73597 & 13842 \\
\hline 20 & -43350 & 21872 & 87094 & 4057 \\
\hline
\end{tabular}




\section{RESEARCH RESULTS}

\section{SPCT Validation Using Successful Project}

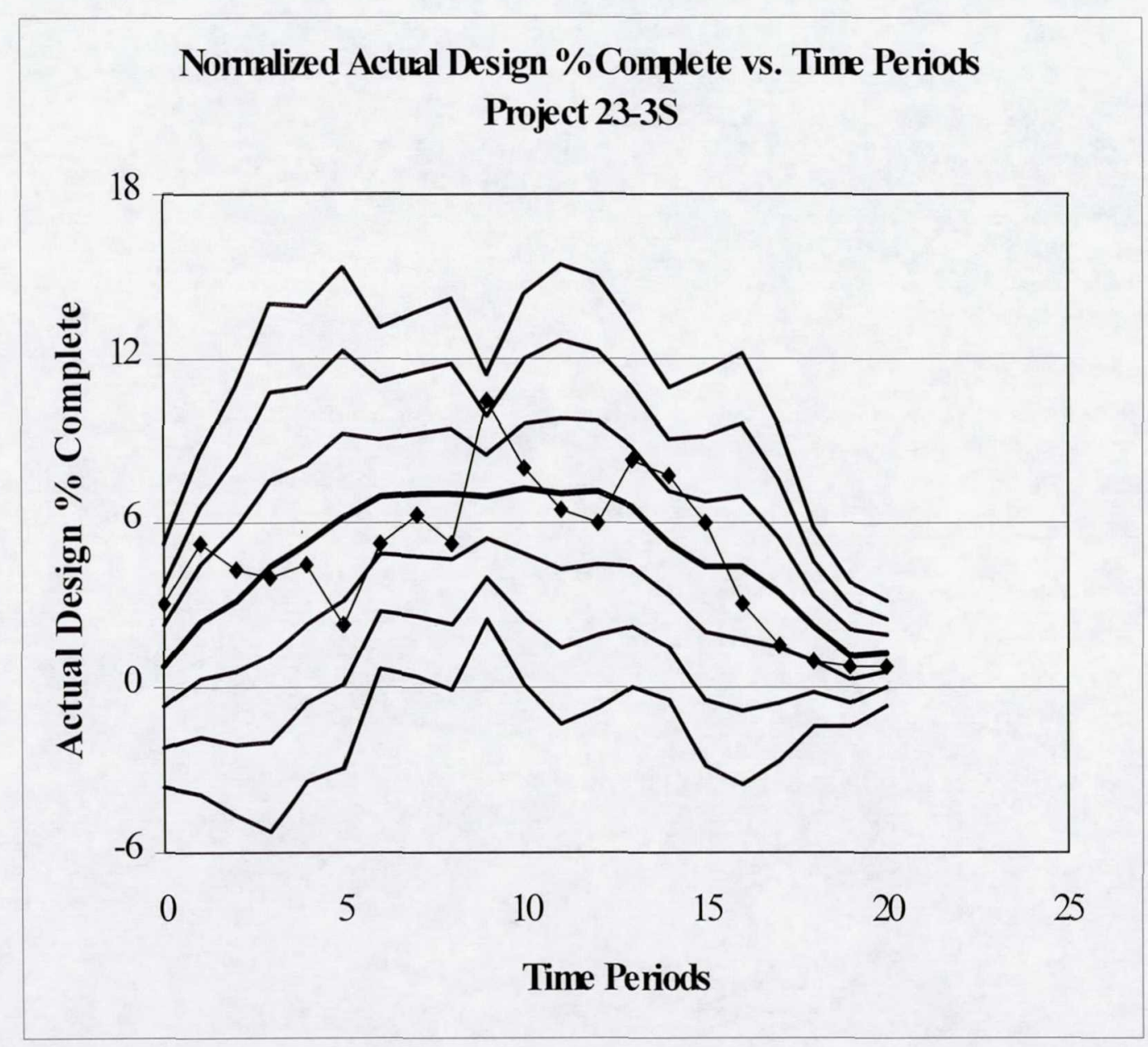

- Process industry

- Pattern in-control per Rule 3m

- Pattern validates control Chart

\begin{tabular}{|c|c|c|c|c|}
\hline IP & LCL & CL & UCL & \\
\hline 0 & -3.63 & 0.80 & 5.24 & \\
\hline 5 & -2.99 & 6.17 & 15.33 & \\
\hline 10 & 0.12 & 7.27 & 14.41 & \\
\hline 15 & -2.83 & 4.37 & 11.56 & \\
\hline & -0.65 & 1.25 & 3.16 & \\
\hline
\end{tabular}




\section{RESEARCH RESULTS \\ SPCT Validation Using Failed Project}

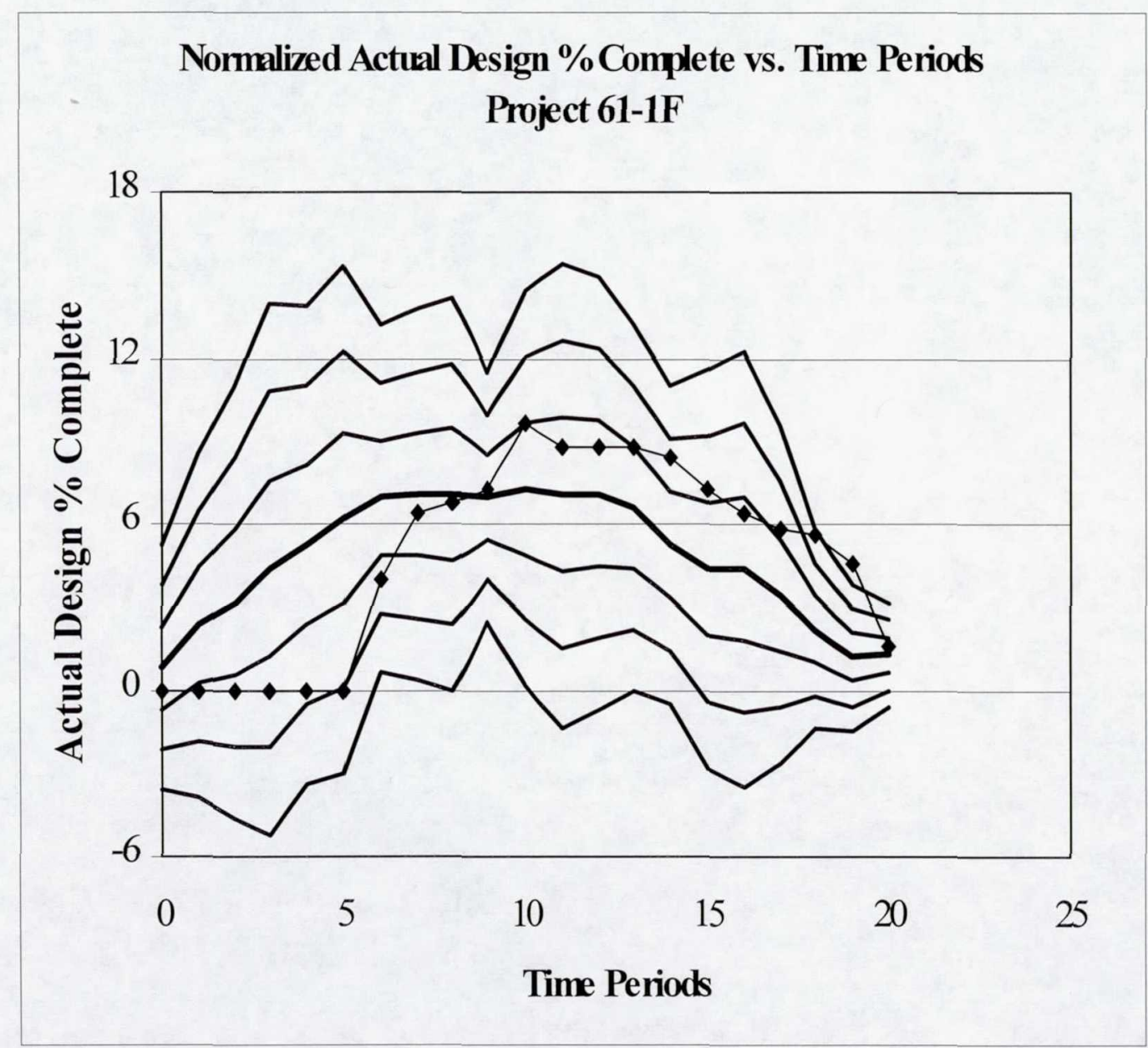

-Process industry

-Out-of-control per Rules 1, 3, \& 6

- Pattern validates control chart

\begin{tabular}{|c|c|c|c|c|}
\hline $\mathrm{P}$ & $\underline{\mathrm{LCL}}$ & $\underline{\mathrm{CL}}$ & UCL & $61-1 \mathrm{~F}$ \\
\hline 0 & -3.63 & $0 . \overline{80}$ & 5.24 & 0.00 \\
\hline & -2.99 & 6.17 & 15.33 & 0.00 \\
\hline 10 & 0.12 & 7.27 & 14.41 & 9.60 \\
\hline 15 & -2.83 & 4.37 & 11.56 & 7.20 \\
\hline & -0.65 & 1.25 & 3.16 & 1.60 \\
\hline
\end{tabular}




\section{RESEARCH RESULTS}

\section{SPCT Validation Using Successful Project}

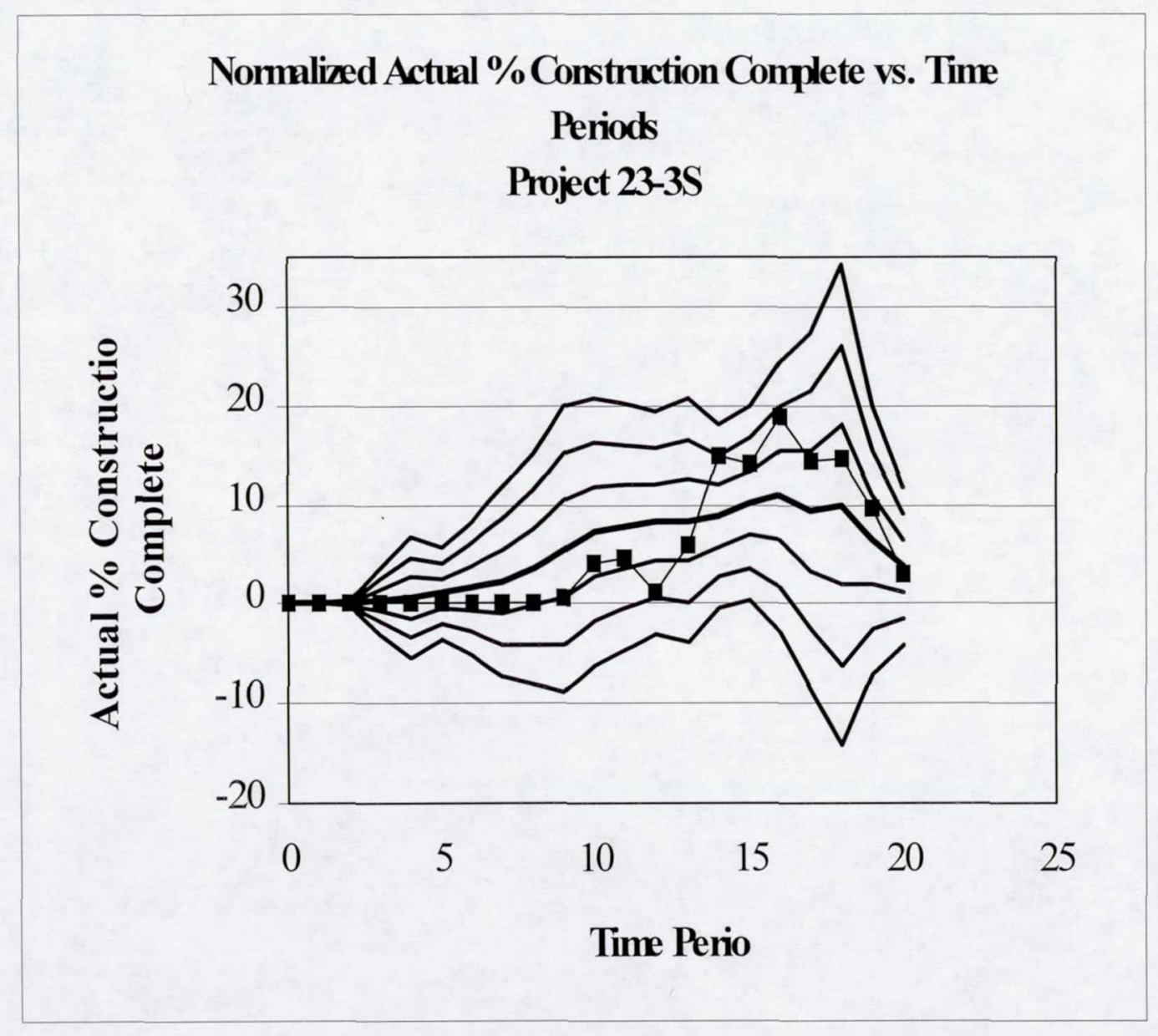

- Process industry

- Pattern in-control per Rule $2 \mathrm{~m}$

- Pattern validates control chart

$\begin{array}{rrrrr}\frac{\mathrm{TP}}{\mathrm{N}} & \underline{\mathrm{LCL}} & \mathrm{CL} & \underline{\mathrm{UCL}} & 23-3 \mathrm{~S} \\ 5 & 0.00 & 0.00 & 0.00 & 0.00 \\ 5 & -3.61 & 1.02 & 5.65 & 0.00 \\ 10 & -6.23 & 7.22 & 20.67 & 4.19 \\ 15 & .41 & 10.16 & 19.90 & 14.20 \\ 20 & -4.01 & 3.82 & 11.65 & 3.02\end{array}$




\section{RESEARCH RESULTS \\ SPCT Validation Using Failed Project}

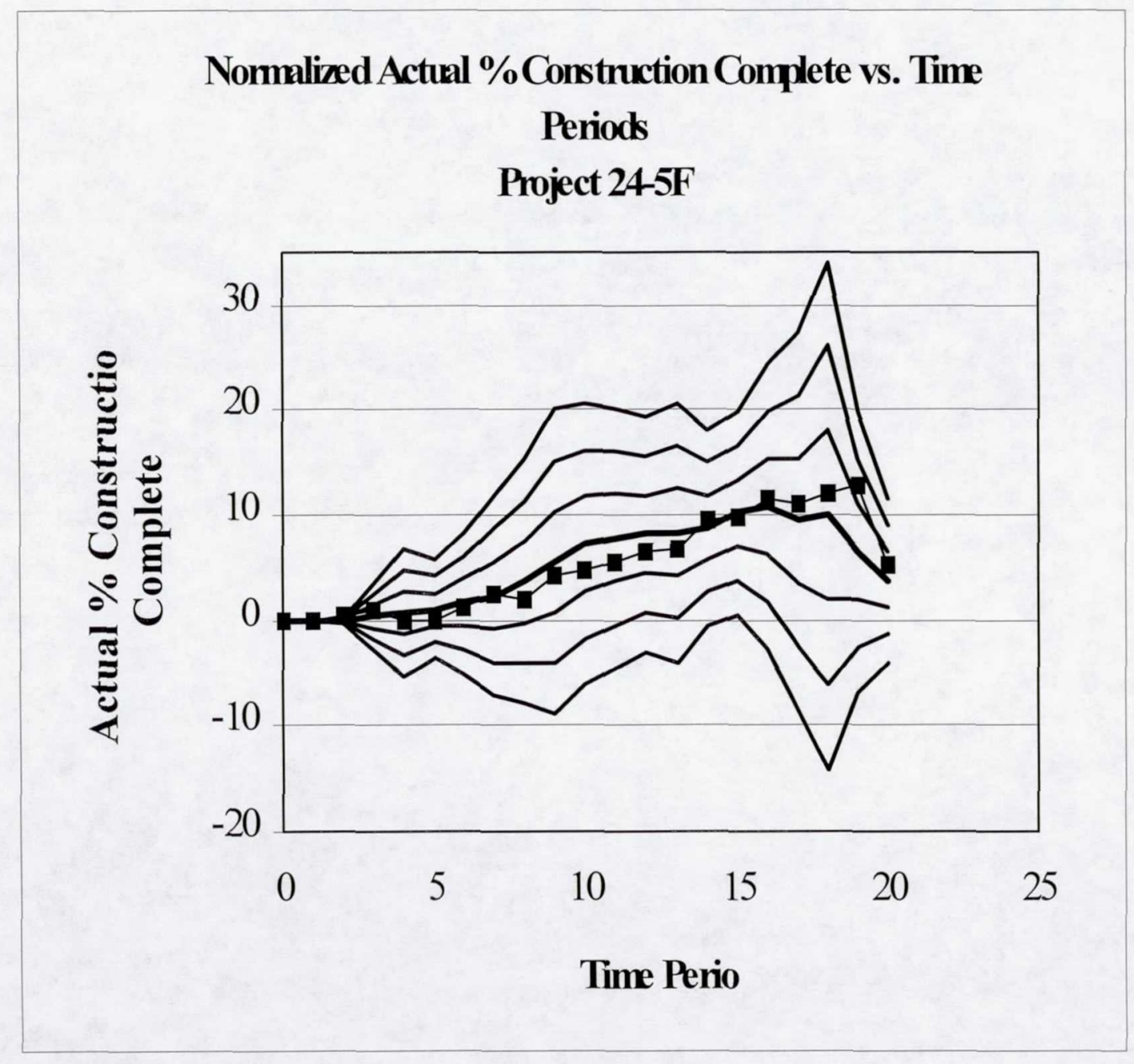

- Process industry

- Out-of-control per Rule 2

- Pattern validates control chart

\begin{tabular}{|c|c|c|c|c|}
\hline TP & $\underline{\mathrm{LCL}}$ & $\underline{\mathrm{CL}}$ & UCL & $24-5 \mathrm{~F}$ \\
\hline 0 & 0.00 & 0.00 & 0.00 & 0.00 \\
\hline 5 & -3.61 & 1.02 & 5.65 & 0.00 \\
\hline 10 & -6.23 & 7.22 & 20.67 & 4.70 \\
\hline 15 & .41 & 10.16 & 19.90 & 9.89 \\
\hline 20 & -4.01 & 3.82 & 11.65 & 5.18 \\
\hline
\end{tabular}




\section{RESEARCH RESULTS}

\section{SPCT Validation Using Successful Project}

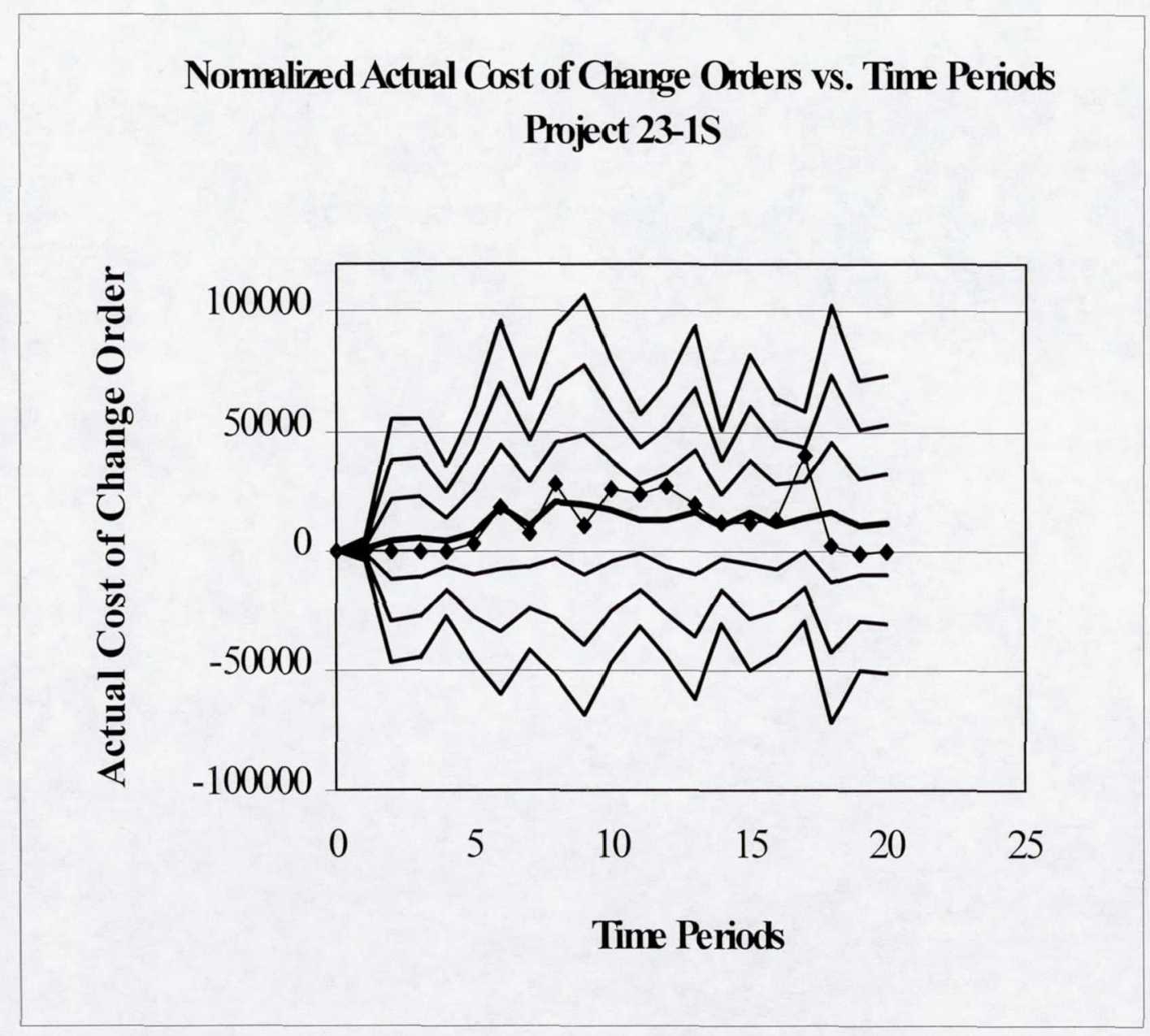

- Power industry

- Pattern in-control

- Pattern validates control chart

$\begin{array}{rrrrr}\frac{\text { TP }}{0} & \underline{\text { LCL }} & \underline{\mathrm{CL}} & \underline{\mathrm{UCL}} & \frac{23-1 \mathrm{~S}}{0} \\ 5 & -45536 & 7549 & 60635 & 2621 \\ 10 & -46625 & 16620 & 79865 & 25403 \\ 15 & -50258 & 15945 & 82149 & 11909 \\ 20 & -51058 & 11182 & 73423 & -725\end{array}$




\section{RESEARCH RESULTS \\ SPCT Validation Using Failed Project}

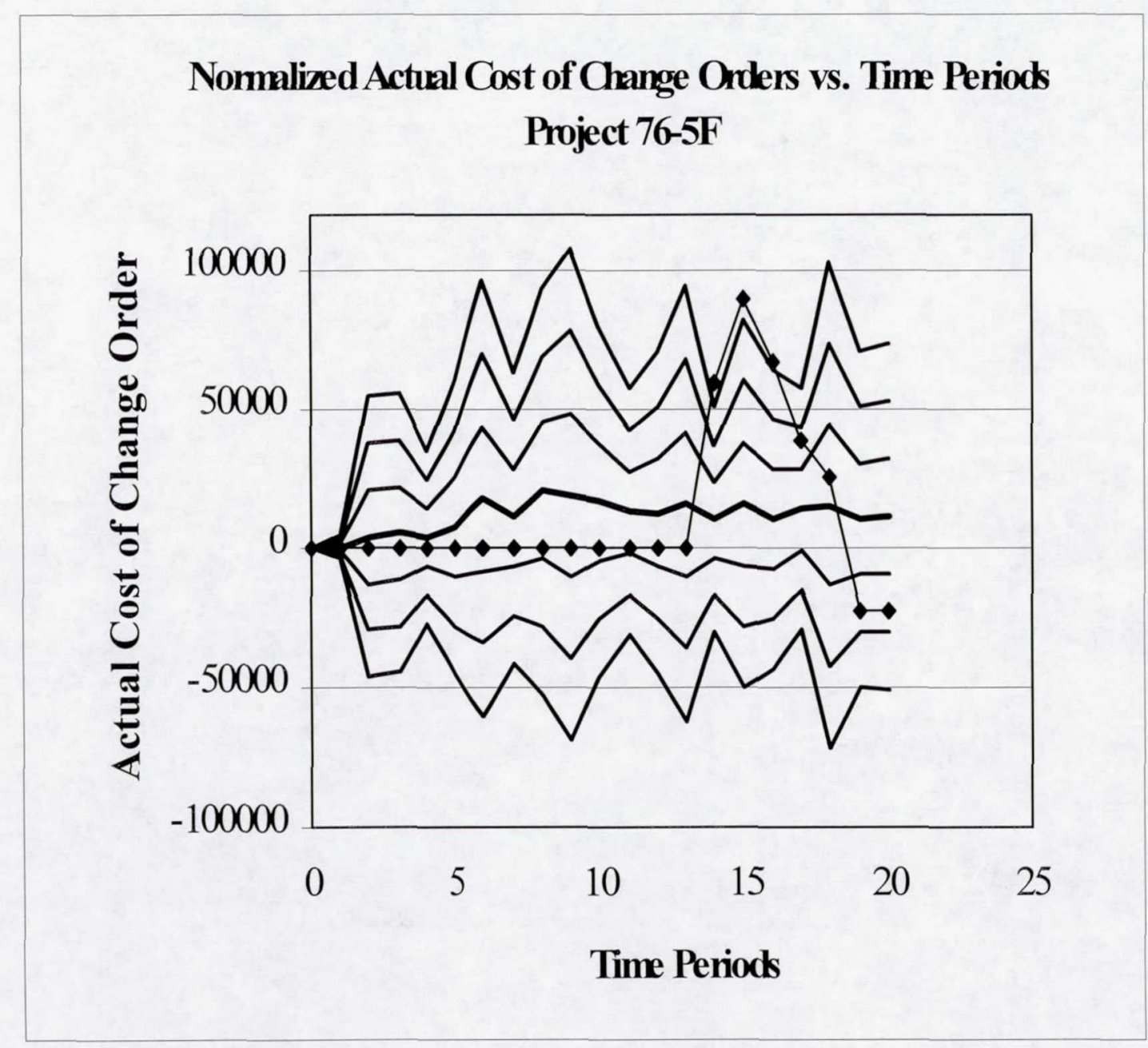

-General Building industry

-Out-of-control per Rules 1 \& 5

-Pattern validates control chart

\begin{tabular}{|c|c|c|c|c|}
\hline TP & $\underline{\mathrm{LCL}}$ & $\underline{\mathrm{CL}}$ & UCL & $76 \underline{-5 \mathrm{~F}}$ \\
\hline 0 & 0 & 0 & 0 & \\
\hline 5 & -45536 & 7549 & 60635 & \\
\hline 10 & -46625 & 16620 & 79865 & \\
\hline 15 & -50258 & 15945 & 82149 & 89643 \\
\hline 20 & -51058 & 11182 & 73423 & -2255 \\
\hline
\end{tabular}




\section{DISCUSSION}

- Check for Normality Assumptions

- Comparison of Project Characteristics

- Control Chart Validation

- Problems Encountered

- Why Control Chart Works 


\section{DISCUSSION \\ Causes for "Out of Control" Patterns}

- Rule 1: One Point Beyond Zone A

-Change in Corp. policy

- Design Change

-Design Step Omitted

- Rule 2: 9 Pts. in a Row in Zone C or Beyond

-New Manager

- New Metrics System Manager

-New Business Rules Instituted 


\section{DISCUSSION}

\section{Causes for "Out of Control" Patterns}

- Rule $3_{1}$ : 6 Pts. in a Row Steadily Increasing

-Poor Team Morale

-Requirements not being met

- Manager or Team Fatigue

-Changes in External Environment

-Emergency or expedition declared

- Rule $3_{2}: 6$ Pts. in a Row Steadily Decreasing

-Opposite causes as in Rule $3_{1}$ 


\section{LIMITATIONS OF STUDY}

- Construction Industry Only

- Limited Amount of Data

- Variables Selected

- Effect of Project Characteristics

- Variable 4 Lack of Normality 


\section{CONTRIBUTION TO THE BODY OF KNOWLEDGE}

- Quantitative Benchmarking Tool

- Dynamic Decision-Making (predictive)

- Industrial Engineering Method

- Environmental Factors

- Quality and Safety 


\section{CONCLUSIONS}

- No Tool Like SPCT Presently Available

- Ratio Method Best

- Cumulative Plot Interpolation

- SPCT Chart Methodology Is Valid

- Can Indicate Health of Project

- May be applied to other industries 


\section{FUTURE RESEARCH}

- Product vs. Process Success

- Study Lower Level Elements

- Study Other Industry Types

- Study Other Project Types 


\section{QUESTIONS}

